\title{
Proposal of ROS-compliant FPGA component for low-power robotic systems (Retraction Notice)
}

Rong Li, Lei Quan, YouLin Cai

Rong Li, Lei Quan, YouLin Cai, "Proposal of ROS-compliant FPGA component for low-power robotic systems (Retraction Notice)," Proc. SPIE 9808, International Conference on Intelligent Earth Observing and Applications 2015, 98082N (9 December 2015); doi: 10.1117/12.2220616

SPIE Event: International Conference on Intelligent Earth Observing and Applications, 2015, Guilin, China 


\section{Proposal of ROS-compliant FPGA component for low-power robotic systems (retraction notice)}

Rong Li, Lei Quan, YouLin Cai

East China Univ. of Technology (China)

Proc. SPIE 9808, International Conference on Intelligent Earth Observing and Applications 2015, 98082N

(December 9, 2015); doi:10.1117/12.2220616

From Conference Volume 9808

International Conference on Intelligent Earth Observing and Applications 2015

Guoqing Zhou; Chuanli Kang

Guilin, China | October 23, 2015

This paper was published on December 9, 2015, and retracted from the SPIE Digital Library on April 14, 2016 , by the publisher upon verification that substantial portions of the paper were copied from the following work without attribution or permission:

Kazushi Yamashina, Takeshi Ohkawa, Kanemitsu Ootsu, and Takashi Yokota, "Proposal of ROS-compliant FPGA Component for Low-Power Robotic Systems - case study on image processing application," 2nd International Workshop on FPGAs for Software Programmers (FSP 2015), London, United Kingdom, September 1, 2015. http://arxiv.org/abs/1508.07123 